\title{
Riscos do uso do Dentifrício Fluoretado na Prevenção e Controle de Cárie na Primeira Infância
}

\author{
Risks of Fluoride Dentifrice in the Prevention and Control of Dental Caries in the Primary Childhood
}

Jaime Aparecido Cury ${ }^{1}$, Lívia Maria Andaló Tenuta ${ }^{2}$

\begin{abstract}
Fluoride toothpaste has been considered responsible for the caries decline which occurred either in developed or in developing countries, such as Brazil. However, since children inadvertently ingest a certain amount of toothpaste while brushing their teeth, there is a concern on the subsequent fluorosis. Nevertheless, the potential risk of fluorosis from fluoride toothpaste has been overestimated based on the dose of fluoride ingested, considering as limit the dose of $0.07 \mathrm{mg} \mathrm{F} / \mathrm{kg}$ body weight/day, and not on the actual fluorosis outcome. The dose of fluoride ingestion from toothpastes has been overestimated because It does not consider how much of fluoride is bioavailable to be absorbed and cause fluorosis. The lack of association between ingestion dose and fluorosis has been shown experimentally and observed epidemiologically by the mild degree of fluorosis found, which enables us to ratify the importance of the recommendation of fluoride toothpaste for caries control in young children, with few concerns on possible side effects.
\end{abstract}

Keywords: fluoride, toothpaste, dental fluorosis

\section{Resumo}

Dentifrício fluoretado tem sido considerado responsável pelo declínio de carie dentária ocorrida tanto em países desenvolvidos quanto nos em desenvolvimento, como o Brasil. Entretanto, como as crianças involuntariamente ingerem certa quantidade de dentifrício quando escovam os dentes, há preocupação com a fluorose decorrente. Entretanto, o risco em potencial do dentifrício provocar fluorose tem sido estimado com base na dose de ingestão de fluoreto, considerando como limite a dose de $0,07 \mathrm{mg} \mathrm{F} / \mathrm{dia} / \mathrm{kg}$ de peso e não na fluorose decorrente. A dose de ingestão de fluoreto por dentifrícios tem sido superestimada, porque não considera quanto do fluoreto ingerido está biodisponível para ser absorvido e provocar fluorose. A falta de associação entre dose de ingestão e fluorose tem sido mostrada experimentalmente e comprovada epidemiologicamente pelo grau não preocupante de fluorose encontrado, possibilitando ratificar a importância da recomendação do uso de dentifrício fluoretado para o controle de cárie, sem grandes preocupações com possíveis efeitos colaterais.

Palavras chave: flúor, fluorose dental, dentifrício
${ }^{1}$ Professor Titular de Bioquímica da Faculdade de Odontologia de Piracicaba, UNICAMP, São Paulo, Brasil

${ }^{2}$ Professora Associada de Bioquímica da Faculdade de Odontologia de Piracicaba, UNICAMP, São Paulo, Brasil

Correspondência: Jaime Aparecido Cury

Endereço: Avenida Limeira 901, UNICAMP, Piracicaba, 13414-903 - Brasil E-mail: jcury@fop.unicamp.br

\section{Introdução}

O declínio de cárie dentária, ocorrido a partir da década de 80 nos países desenvolvidos e a partir dos anos 90' nos em desenvolvimento como o Brasil, tem sido explicado pelo amplo uso de cremes dentais fluoretados (BRATTHALL; HANSEL-PETERSSON; SUNDBERG, 1996; CURY et al., 2004).

Por outro lado, a constatação de que, paralelamente, ocorreu no EUA um aumento da prevalência de fluorose dentária, desencadeou a realização de pesquisas em todo mundo com a preocupação de determinar o risco que o uso abrangente de dentifrício fluoretado poderia provocar nas populações expostas. Essa preocupação no EUA, país que já convive naturalmente com a fluorose devido ao efeito sistêmico da exposição à água fluoretada, fazia sentido e serviu de alerta para outros países como o Brasil, que elegeu a fluoretação da água como meio coletivo de uso de fluoreto para controlar cárie na sua população. Em países europeus, onde a população está exposta a dentifrício fluoretado, a maior prevalência de fluorose é observada onde há água fluoretada (COCHRAN et al., 2004). Em acréscimo, em crianças australianas expostas a água e cremes dentais fluoretados durante os três primeiros anos de vida, o risco de apresentar fluorose é duas vezes maior pela exposição à água que ao dentifrício (DO; SPENCER, 2007).

Entretanto, esse assunto com relação aos dentifrícios fluoretados não tem sido tratado com base nas melhores evidências científicas disponíveis e a prova de isso é a falta de consenso entre instituições e países quanto às recomendações do uso de dentifrícios fluoretados na $1^{\text {a }}$ infância (DOS SANTOS; NADANOVSKY; DE OLIVEIRA, 2010, 2011). Assim, por exemplo, considerando que crianças ingerem fluoreto quando escovam os dentes com dentifrício fluoretado e esse é fator de risco de fluorose (MASCARENHAS; BURT, 1998), e com base em uma única publicação sugerindo a maior contribuição do dentifrício para a dose total de fluoreto ingerido em uma região de água fluoretada (DE ALMEIDA; DA SILVA CARDOSO; BUZALAF, 2007), a Academia Européia de Odontopediatria recomenda que crianças nas faixas etárias de 6 a 24 meses, 2 a 6 anos e aquelas maiores que 6 utilizem respectivamente cremes dentais com 500, 1000 e 1450 ppm F (EUROPEAN ACADEMY OF PAEDIATRIC DENTISTRY, 2009). Essa recomendação, além de não estar baseada nos benefícios anticárie dos dentifrícios fluoretados (WALSH et al., 2010; DOS SANTOS; NADANOVSKY; DE OLIVEIRA, 2012), como discutido em outro artigo dessa série (TENUTA; CURY, 2012), também não está suportada no efeito da fluorose encontrada em populações expostas ao dentifrício isoladamente ou quando combinado com o já conhecido efeito da água fluoretada (FRAZÃO; PERES; CURY, 2011). Do mesmo modo, 
não está suportada em evidência a recomendação de que crianças só devem usar creme dental fluoretado após os 3 anos de idade, pois esta também se baseia em dose de ingestão e não no efeito de fluorose resultante (WONG et al., 2010).

$\mathrm{Na}$ realidade, a grande maioria dos trabalhos sobre a relação de creme dental fluoretado e fluorose está baseado na dose de ingestão de fluoreto por via oral e não no fluoreto biodisponível, isto é na fração que absorvida no trato gastro-intestinal, circula pelo sangue e atinge o esmalte em formação provocando fluorose. Essa superestimação da dose de ingestão de fluoreto pelos cremes dentais e risco de fluorose tem sido usada de forma alarmista em prejuízo dos seus benefícios no controle de cárie, sendo que apenas dois trabalhos tentaram relacionar dose de ingestão com a fluorose decorrente (MARTINS et al., 2008; WARREN et al., 2009). Deve ser ressaltado já aqui nessa introdução que a dose de $0,07 \mathrm{mg} \mathrm{F} / \mathrm{dia} / \mathrm{kg}$ de peso, considerada como limite de ingestão de fluoreto por uma criança, foi estimada (BURT, 1992) quando o mecanismo de ação local do fluoreto no controle de cárie não estava consolidado e fluoreto era considerado um micronutriente necessário.

Assim, o objetivo desse artigo será uma abordagem sobre os riscos do uso de creme dental fluoretado na $1^{\mathrm{a}}$ infância com base no conhecimento científico do metabolismo do fluoreto, no mecanismo de desenvolvimento da fluorose dentária, na estimada dose de risco de fluorose e no significado clínico da fluorose decorrente.

\section{Fatores que interferem com a toxicidade do fluoreto}

O conhecimento sobre o metabolismo do fluoreto (íon flúor, $\mathrm{F}^{-}$) é fundamental para entender a toxicidade do fluoreto, quer seja a aguda como a crônica. Toxicidade aguda diz respeito à ingestão de uma grande quantidade de fluoreto de uma única só vez e a crônica quando pequenas quantidades são ingeridas diariamente durante prolongados períodos de tempos. Por outro lado, para provocar toxicidade o fluoreto ingerido por via oral precisa ser absorvido, dando sustentação à afirmação "qualquer fluoreto (íon flúor) ingerido por via oral e absorvido pelo organismo terá possibilidade de manifestar algum efeito colateral" (TENUTA; CURY, 2009).

Diversos fatores interferem com a absorção gastro-intestinal de fluoreto, como:

a) A solubilidade do sal de fluoreto: Para ser absorvido o fluoreto precisa estar solúvel e assim enquanto todo o $\mathrm{F}^{-}$do $\mathrm{NaF}$ (solubilidade $=5 \%$ ) é passível de ser absorvido, a do $\mathrm{CaF}_{2}$ é extremamente limitada porque a solubilidade do fluoreto de cálcio é de apenas 0,0015\% (3.000 vezes menor). Assim, nos cremes dentais contendo $\mathrm{Ca}^{++}$como abrasivo, parte do $\mathrm{F}^{-}$presente está insolúvel dentro do tubo de dentifrício (CONDE; RABELO; CURY, 2003), e assim quando esse creme dental é ingerido nem todo o fluoreto será absorvido, isto é, não está $100 \%$ biodisponível. Esse conhecimento de biodisponibilidade de drogas em geral quando ingeridas por via oral é antigo e é válido para cremes dentais (ROLDI; CURY, 1986; DRUMMOND; CURZON; STRONG, 1990; FALCÃO; TENUTA; CURY, 2012).

A figura 1 mostra a concentração de fluoreto no sangue de voluntários após a ingestão experimental de creme dental contendo ou não cálcio no abrasivo (ROLDI; CURY, 1986). Essa redução de absorção de $\mathrm{F}^{-}$a partir do dentifrício contendo $\mathrm{Ca}$ é coerente com a concentração de fluoreto solúvel encontrada nesses cremes dentais e foi metabolicamente confirmada pela menor excreção de $\mathrm{F}^{-}$na urina dos voluntários (ROLDI; CURY, 1986).

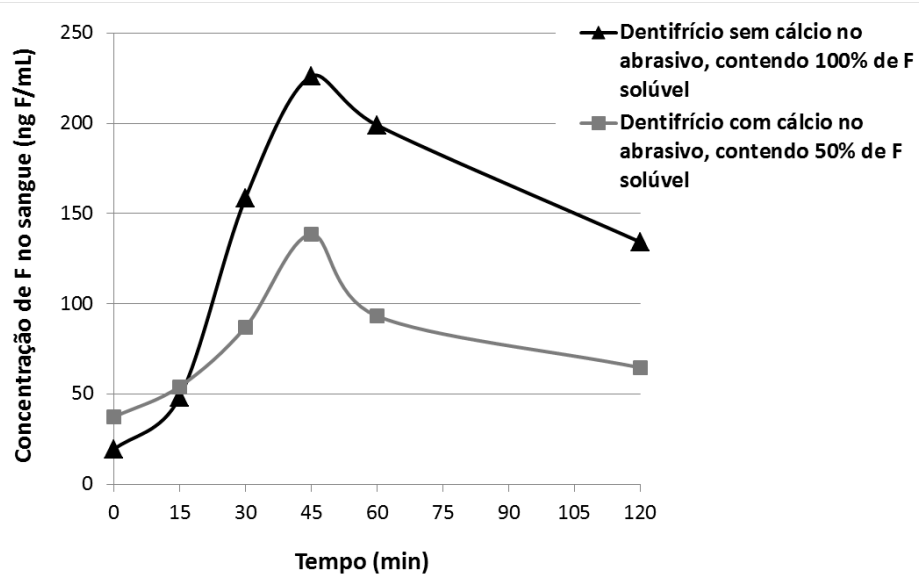

Figura 1: Biodisponibilidade do fluoreto (fluoreto absorvido) após a ingestão de dentifrícios contendo ou não cálcio no abrasivo, de acordo com a concentração de F solúvel. Média ( $n=3$ ) (ROLDI; CURY, 1986).

Formulações contendo Ca no abrasivo são as mais vendidas no Brasil, nas quais $20-30 \%$ do fluoreto total presente não está solúvel (RICOMINI FILHO et al., 2012) para ser absorvido. Esses cremes dentais são formulados com $\mathrm{MFP} / \mathrm{CaCO}_{3}$ tendo $1.450 \mathrm{ppm}$ de flúor total, porém aproximadamente 1.000-1.100 ppm de flúor solúvel. Em contraste, cremes dentais não contendo $\mathrm{Ca}$ no abrasivo são geralmente formuladas com sílica $\left(\mathrm{SiO}_{2}\right)$ e $\mathrm{NaF}$ tendo $1.100 \mathrm{ppm}$ de flúor total, do qual todo ele está solúvel.

Cremes dentais contendo $\mathrm{NaF} / \mathrm{SiO}_{2}$ (1100 ppm F) são no Brasil os mais usados por crianças de maior nível socioeconômico e os contendo $\mathrm{MFP} / \mathrm{CaCO}_{3}$ (1450 ppm F) são os usados pelas crianças de menor nível socioeconômico porque são as usadas por toda a família (MARTINS et al., 2011).

A figura 2, mostra pelas curvas de absorção que quando uma mesma quantidade de flúor total é ingerido por esses 2 tipos de cremes dentais, a biodisponibilidade daquele contendo $\mathrm{MFP} / \mathrm{CaCO}_{3}$ é menor (FALCÃO; TENUTA; CURY, 2012) porque só o solúvel está biodisponível. Logo, embora o creme dental com $\mathrm{MFP} / \mathrm{CaCO}_{3}$ apresente uma concentração de fluoreto total $30 \%$ maior $(1500 \mathrm{ppm}$ F) do que o com $\mathrm{NaF} / \mathrm{SiO}_{2}$ (1100 ppm F), o risco de toxicidade será igual quando da ingestão. Isso é relevante porque embora o primeiro seja de menor custo, como a concentração total é maior tem havido restrição ao seu uso por crianças, agravado por trabalhos que consideram a concentração total nos cálculos de dose de ingestão, superestimando o risco e a porcentagem de contribuição para a dose total de ingestão em comparação com a dieta, como será discutido na sessão pertinente nesse artigo.

b) Conteúdo gástrico: Quando o fluoreto está solúvel e é ingerido, estando a pessoa em jejum (estômago vazio), todo o fluoreto é absorvido, porém se ingerido até 15 min após uma refeição apenas uma porcentagem será absorvida (EKSTRAND; SPAK; VOGEL, 1990). Isso é valido quando creme dental fluoretado é ingerido e experimentalmente foi demonstrado que a quantidade absorvida foi 20 e $40 \%$ menor respectivamente quando da ingestão após o café da manhã e refeição, tipicamente brasileiras (CURY et al., 2005). Nesse trabalho também ficou comprovado que o efeito sistêmico da ingestão de um dentifrício com 550 ppm $\mathrm{F}$ com o estômago vazio seria o mesmo de um com 1.100 ppm $F$, porém ingerido logo após a refeição, contrastando com a recomendação de que dentifrício de baixa concentração de fluoreto seria uma alternativa para diminuir o risco de fluorose, mas dando suporte a discussão sobre esse assunto (Paiva et al., 2003). 


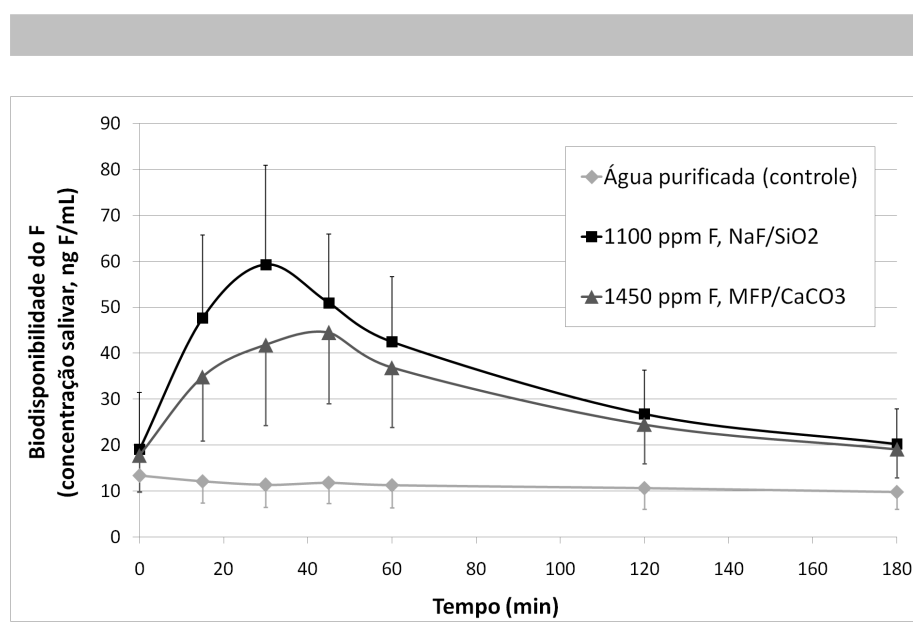

Figura 2: Biodisponibilidade do fluoreto (fluoreto absorvido) após a ingestão de uma mesma quantidade de fluoreto a partir de dentifrícios contendo $100 \%$ do fluoreto biodisponível (1100 ppm F, NaF/SiO2) ou aproximadamente $80 \%$ do fluoreto biodisponível (1450 ppm $F$, MFP/CaCO3). Média \pm desvio padrão, $n=20$ (FALCÃO; TENUTA; CURY, 2012).

c) Ílons interferentes: Cátions como $\mathrm{Ca}^{++}, \mathrm{Al}^{+++}$e $\mathrm{Mg}^{++}$ reduzem a absorção gastrointestinal de fluoreto se estiverem presentes no estômago quando da ingestão de fluoreto (WHITFORD, 1996). Assim, além da presença de alimentos no estomago reduzirem mecanicamente a absorção de fluoreto, se estes contiverem $\mathrm{Ca}^{++}$haverá um efeito físicoquímico adicional.

d) $\mathbf{p H}$ : A absorção de fluoreto é facilitada em $\mathrm{pH}$ baixo devido à formação de HF que se difunde passivamente pela mucosa gástrica e na primeira parte do intestino delgado (WHITFORD; PASHLEY, 1984). Os dentifrícios a base de $\mathrm{MFP} / \mathrm{CaCO}_{3}$ tem $\mathrm{pH}$ alcalino e os a base de sílica, neutro, mas não é conhecido se isso se reflete em diferença de absorção de fluoreto entre essas formulações.

A toxicidade do fluoreto ingerido por via oral depende principalmente da fração desse fluoreto que é absorvido no trato gastro-intestinal, mas também deve ser considerado o quanto desse fluoreto é excretado pela urina. Assim, a eliminação do fluoreto circulante depende da função renal e $\mathrm{opH}$ da urina influencia na excreção renal do fluoreto, pois quando de $\mathrm{pH}$ ácido o fluoreto é reabsorvido nos túbulos renais e retorna para o sangue mantendo o nível metabólico alto. Assim, acidose respiratória ou metabólica pode agravar a fluorose quando da ingestão de fluoreto durante a formação dos dentes.

\section{Toxicidade aguda do fluoreto e dentifrício fluoretado}

Quando altas quantidades de fluoreto são ingeridas poderão ocorrer desde sintomas gastro-intestinais, como náuseas, vômitos e diarréia, até parada cardiorespiratória e morte (CURY; TENUTA 2011). Tendo em vista acidentes fatais quando pessoas foram submetidas a doses de fluoreto menores que as chamadas de dose certamente letal e seguramente tolerada, o parâmetro hoje aceito é chamado de dose provavelmente tóxica (DPT) a qual corresponde a dose de ingestão de $5,0 \mathrm{mg} \mathrm{F} / \mathrm{kg}$ de peso corpóreo da vítima (WHITFORD, 1992). De acordo com esse parâmetro, em nenhum procedimento clínico ou nenhum produto de auto-uso de fluoreto pode sujeitar o paciente ou usuário a DPT. Assim, acidentes letais já ocorreram por ingestões de comprimidos (suplementos) medicamentosos contendo fluoreto, mas creme dental fluoretado pode ser considerado seguro quanto à toxicidade aguda por fluoreto (tabela 1).

A tabela 1 mostra quanto de creme dental crianças de idades entre 1 a 6 anos teriam que ingerir de dentifrício de 1.000 ou $1.500 \mathrm{ppm} \mathrm{F} \mathrm{(mg} \mathrm{F/kg)} \mathrm{para} \mathrm{serem} \mathrm{submetidas} \mathrm{a} \mathrm{DPT} \mathrm{de} \mathrm{5,0} \mathrm{mg} \mathrm{F/kg.}$

Tabela 1. Quantidade (g) de dentifrício de 1.000 ou 1.500 ppm F que crianças de idade de 1 a 6 anos (10 a $21 \mathrm{~kg}$ ) teriam que ingerir para serem submetidas a DPT.

\begin{tabular}{cccc}
$\begin{array}{c}\text { Idade } \\
\text { (anos) }\end{array}$ & $\begin{array}{c}\text { Peso da Criança } \\
(\mathrm{kg})\end{array}$ & \multicolumn{2}{c}{ Dentifrício (ppm F) } \\
\cline { 3 - 4 } & & 1.000 & 1.500 \\
\hline 1 & 10 & 50 & 33 \\
2 & 12 & 60 & 40 \\
3 & 15 & 75 & 50 \\
4 & 17 & 85 & 57 \\
5 & 19 & 95 & 63 \\
6 & 21 & 105 & 70 \\
\hline
\end{tabular}

Assim, uma criança de idade de 2 anos pesando $12 \mathrm{~kg}$ teria que ingerir de uma única só vez $60 \mathrm{~g}$ de um dentifrício de concentração de 1.000 ppm F para ser submetida a DPT de $5,0 \mathrm{mg}$ $\mathrm{F} / \mathrm{kg}$. Isso equivale por exemplo a ingerir $55 \mathrm{~g}$ do creme dental Tandy (1.100 ppm F), o qual é maior que o peso contido em um tubo desse dentifrício $(50 \mathrm{~g})$. A probabilidade de ocorrer esse acidente é remota e embora haja relatos que crianças comam creme dental, não há casos reportados na literatura sobre intoxicação aguda por fluoreto a partir do uso indevido de creme dental fluoretado. A não ingestão de grandes quantidades de creme dental é plausível, porque sendo produtos de higiene bucal, eles contém de 0,5 a $1 \%$ de detergente 0 qual deverá provocar mal estar na criança antes de ser atingida a DPT.

Entretanto, até que as crianças não adquiram o reflexo de cuspir elas acabam ingerindo diariamente certa quantidade de creme dental quando escovam seus dentes, as submetendo a toxicidade crônica de exposição a fluoreto, como será abordado a seguir.

\section{Toxicidade crônica do fluoreto, com ênfase a fluorose dental e efeito de dentifrício}

Quando quantidades pequenas de fluoreto são ingeridas diariamente durante a formação dos dentes, estes estão sujeitos ao fenômeno de fluorose dental. O entendimento de como o fluoreto provoca fluorose dental é importante porque: a) Facilita entender porque, em doses devido a água otimamente fluoretada ou a dentifrício fluoretado, ele não provoca fluorose óssea; b) Auxilia no diagnóstico diferencial entre opacidades fluoróticas de outros defeitos de formação do esmalte; c) Ajuda entender porque o tempo de duração de uma certa dose de exposição é mais importante que "janela de risco de fluorose" ou picos de doses maiores; d) Possibilita entender que seu efeito não é celular no ameloblasto, mas sim extracelularmente na matriz do esmalte em formação; d) Ajuda a entender porque o efeito depende da fração do fluoreto ingerido que é absorvido e não da dose de ingestão; e) Facilita entender porque dentes homólogos ou formados no mesmo período são igualmente afetados pela fluorose; f) Possibilita entender porque a fluorose é mais perceptível quando o dente é seco.

Com relação a fatores de risco, água fluoretada na concentração ótima, suplementos pós-natal de flúor, fórmulas infantis 
à base de soja e cremes dentais fluoretados têm sido associados com fluorose dental (MASCARENHAS, 2000).

Entretanto, à semelhança da toxicidade aguda, para a qual a dose limite de 5,0 mg F/kg (DPT) é utilizada como parâmetro de segurança, com relação a fluorose dental tem sido sugerido que 0,05 a $0,07 \mathrm{mg} \mathrm{F} / \mathrm{kg}$ peso corpóreo/dia deve ser a dose máxima aceitável para que "a fluorose não provoque no esmalte alterações que comprometam a estética dental". Como toda a discussão atual da associação entre fluorose e dentifrício está centrada nessa dose de ingestão de fluoreto, em primeiro lugar a gênese dessa dose deve ser conhecida para conhecer suas limitações.

Na realidade, a gênese dessa dose é empírica (BURT, 1992) e ela foi estabelecida quando era aceito que o efeito do fluoreto no controle de cárie seria sistêmico, sendo indispensável ingerir fluoreto durante a formação dos dentes para resistência a cárie. Assim, a semelhança do estabelecimento de uma concentração 'ótima' de fluoreto na água de beber, foi estabelecido que a dose de 0,05 a 0,07 $\mathrm{mg} \mathrm{F/kg}$ peso corpóreo/dia seria considerada 'ótima' em termos do balanço entre o benefício anticárie do fluoreto ingerido e seu risco de fluorose dental, porém essa chamada "dose ótima" tem sido questionada (WARREN et al. 2009).

Por outro lado, o estabelecimento de uma dose de risco é relevante porque fluorose dental é um efeito sistêmico, portanto o grau de fluorose provocado nos dentes dependerá da concentração de $\mathrm{F}^{-}$no sangue, a qual depende da dose de ingestão diária de fluoreto (FEJERSKOV; MANJI; BAELUM, 1990). Entretanto, a maioria das publicações sobre o potencial de risco de dentifrício fluoretado em termos de fluorose apenas considera a dose de ingestão de $0,07 \mathrm{mg}$ $\mathrm{F} / \mathrm{kg} /$ dia como a máxima aceitável, sem relacionar dose com o desfecho de fluorose decorrente. Na realidade, desde que essa dose empírica foi estabelecida há mais de 20 anos, somente dois trabalhos longitudinais foram feitos relacionando a dose que crianças foram submetidas na idade de 1-3 anos e a consequente fluorose na dentição permanente (MARTINS et al., 2008, WARREN et al., 2009).

Um desses estudos foi realizado no Brasil com 71 crianças de 24 a 30 meses de idade que viviam em duas cidades abastecidas por água fluoretada e estavam usando dentifrício fluoretado (PAIVA; LIMA; CURY, 2003). Elas estavam sendo submetidas à dose total de $0,09 \mathrm{mg} \mathrm{F} / \mathrm{kg} /$ dia $(60 \%$ devido a ingestão de dentifrício e $40 \%$ pela dieta) e a fluorose decorrente dessa dose foi avaliada quando os incisivos centrais permanentes dessas crianças estavam totalmente irrompidos. O trabalho mostrou (MARTINS et al., 2008) que apesar da dose média de exposição diária ao fluoreto estar $30 \%$ acima do máximo empiricamente aceitável de $0,07 \mathrm{mg} \mathrm{F} / \mathrm{kg} / \mathrm{dia}, 41 \%$ das crianças examinadas não apresentaram nenhum grau de fluorose. Das $59 \%$ que apresentavam fluorose, $90 \%$ delas tiveram fluorose grau 1, a qual não compromete a estética dental e a qualidade de

vida dos acometidos (CHANKANKA et al., 2010). Assim, não foi encontrada associação entre dose total de ingestão de fluoreto, dose pela dieta ou dose pelo dentifrício com a fluorose decorrente, sugerindo que o uso da dose de $0,7 \mathrm{mg} \mathrm{F} / \mathrm{kg} /$ dia nas recomendações de uso de flúor em termos de fluorose não está baseada em evidência de relação dose-efeito, sendo esse resultado confirmado em outro estudo feito no EUA (WARREN et al., 2009).

Além da discussão atual sobre fluorose estar centrada nessa estimada dose de risco de $0,07 \mathrm{mg} \mathrm{F} / \mathrm{kg}$ de peso corporal/dia, a prevalência de fluorose dental encontrada em crianças expostas somente a dentifrício fluoretado é menor do que a esperada com base na dose de ingestão de dentifrício (RICHARDS; BANTING, 1996), sugerindo que esta dose está sendo superestimada. Há pelo menos 3 explicações para essa superestimação:

1- A primeira está baseada no cálculo de quanto fluoreto uma criança ingere por dia ao escovar os dentes. A quantidade de dentifrício colocado na escova é pesada e conhecendo sua concentração de fluoreto, calcula-se quanto de fluoreto está sendo usado para escovar os dentes. Tudo que a criança cuspir é colocado num frasco junto com a escova tendo resíduos de dentifrício. A quantidade de fluoreto recuperada nesse frasco representa a quantidade não ingerida pela criança, a qual subtraída da quantidade colocada na escova fornece quanto de fluoreto foi ingerido em uma escovação. A seguir é perguntado para os pais ou responsáveis quantas vezes por dia a criança escova os dentes. Assim, a quantidade de fluoreto encontrada experimentalmente quando de uma escovação é multiplicada pelo número de escovações diárias relatadas e dividida pelo peso da criança, sendo estimada a dose de ingestão, a qual que pode estar inflacionada pela frequência de escovação relata (MARTINS et al., 2011).

2- Outra explicação, como já descrito, está relacionada ao tempo decorrente entre a ingestão de alimentos e a escovação dental. Assim, se os dentes forem escovados até 15 min após as refeições nem todo fluoreto ingerido dentifrício será absorvido (EKSTRAND; SPAK; VOGEL, 1990; CURY et al., 2005), enfatizando a importância de que as crianças escovem seus dentes logo após as refeições.

3- A terceira explicação, também já parcialmente abordada, diz respeito à composição dos dentifrícios, pois naqueles tendo cálcio no abrasivo, parte do fluoreto está insolúvel e assim nem todo fluoreto ingerido é absorvido. Esse fato é relevante porque a dose a qual crianças são submetidas por dentifrícios contendo cálcio no abrasivo tem sido superestimada na comparação com dentifrícios à base de sílica. Assim, quando do cálculo da dose de ingestão de fluoreto por dentifrícios deve ser considerada a concentração de fluoreto solúvel no creme dental e não o total, como tem sido erroneamente feito na maioria dos estudos feitos sobre esse assunto. Comprovando essa preocupação com superestimação de dose, a tabela 2 mostra as concentrações de fluoreto em dentifrícios usados por crianças brasileiras (OLIVEIRA et al., 2012). Nos dentifrícios chamados de 'familiares' nem todo fluoreto está solúvel para ser absorvido, mas naqueles chamados de 'infantis' todo fluoreto está biodisponível.

Tabela 2. Concentração (ppm F) de fluoreto total (FT) declarado na embalagem, FT encontrado na análise laboratorial e fluoreto total solúvel (FTS) encontrado nos cremes dentais, de acordo com as pastas usadas pelas crianças (Media $\pm d p ; n$ )

\begin{tabular}{|c|c|c|c|}
\hline \multirow[b]{2}{*}{$\begin{array}{l}\text { Cremes } \\
\text { Dentais }\end{array}$} & \multicolumn{3}{|c|}{ Concentração (ppm F) } \\
\hline & $\begin{array}{c}\text { FT } \\
\text { declarado }\end{array}$ & $\begin{array}{c}\text { FT } \\
\text { encontrad } \\
0 \\
\end{array}$ & $\begin{array}{c}\text { FTS } \\
\text { encontrad } \\
0 \\
\end{array}$ \\
\hline $\begin{array}{l}\text { Familiares* } \\
\quad(n=80)\end{array}$ & $1424.3 \pm 12.0$ & $\begin{array}{c}1434.0 \pm \\
16.2\end{array}$ & $971.6 \pm 23.4$ \\
\hline $\begin{array}{l}\text { Infantis** } \\
(n=79)\end{array}$ & $1074.9 \pm 13.1$ & $\begin{array}{c}1062.3 \pm \\
12.4\end{array}$ & $1070.7 \pm 24.5$ \\
\hline $\begin{array}{l}\text { Todos } \\
(n=159)\end{array}$ & $1251.8 \pm 14.6$ & $\begin{array}{c}1250.5 \pm \\
18.0\end{array}$ & $1020.0 \pm 17.3$ \\
\hline
\end{tabular}

*Usado por toda a família; **usado exclusivamente pelas crianças

Assim, como mostra a tabela 3 , se no cálculo de dose de ingestão for considerado o flúor total do dentifrício, o dentifrício 'familiar' teria maior risco de provocar fluorose que o 'infantil'. Porém, considerando apenas o solúvel, eles seriam equivalentes. Esse fato é extremamente relevante no Brasil porque os dentifrícios 'familiares' 
formulados a base de $\mathrm{MFP} / \mathrm{CaCO}_{3}$ custam até $50 \%$ menos que os 'infantis' a base de NaF/sílica.

Tabela 3. Dose estimada de ingestão de fluoreto $(\mathrm{mg} \mathrm{F} / \mathrm{dia} / \mathrm{kg}$ peso corporal) a qual crianças seriam submetidas considerando o fluoreto total(FT) declarado no tubo de pasta, e FTe fluoreto total solúvel (FTS) encontrado nas analises, de acordo com os cremes dentais usados (Media $\pm d p ; n$ )

\begin{tabular}{cccc}
\hline & \multicolumn{2}{c}{ Dose (mg F/dia/kg), considerando } \\
\cline { 2 - 4 } $\begin{array}{c}\text { CremesDe } \\
\text { ntais }\end{array}$ & $\begin{array}{c}\text { FT } \\
\text { declarado }\end{array}$ & $\begin{array}{c}\text { FT } \\
\text { encontra } \\
\text { do }\end{array}$ & $\begin{array}{c}\text { FTS } \\
\text { encontrado }\end{array}$ \\
\hline $\begin{array}{c}\text { Familiares } \\
\text { ( } n=80)\end{array}$ & $0.074 \pm$ & $0.074 \pm$ & $0.039 \pm 0.005 \mathrm{~A}, \mathrm{~b}$ \\
Infantis** & $0.007 \mathrm{~A}, \mathrm{a}$ & $0.007 \mathrm{~A}, \mathrm{a}$ & $0.039 \pm 0.005 \mathrm{~A}, \mathrm{a}$ \\
$(\mathrm{n}=79)$ & $0.007 \mathrm{~B}, \mathrm{a}$ & $0.003 \mathrm{~B}, \mathrm{a}$ & $0.039 \pm 0.005$ \\
Todos & $0.057 \pm$ & $0.057 \pm$ & $0.039 \pm 0.003 \mathrm{~b}$ \\
$(\mathrm{n}=159)$ & $0.004 \mathrm{a}$ & $0.004 \mathrm{a}$ & \\
\hline
\end{tabular}

*Usados por toda a família; **usado exclusivamente pelas crianças. Médias seguidas por letras distintas diferem estatisticamente $(p<0.05)$; letras maiúsculas entre tipos de creme dental (valores nas colunas) e minúsculas para cada creme dental (valores nas linhas).

Essa superestimação da dose de ingestão de fluoreto por dentifrícios está de acordo com os resultados de prevalência de fluorose dental encontrados no último levantamento nacional de saúde bucal (BRASIL, 2010). Como mostra a figura 3, a maior prevalência de fluorose no Brasil é de $15 \%$ de fluorose muito leve e leve, a qual não afeta a qualidade de vida das pessoas (CHANKANKA et al., 2010). Esse dado nacional confirma dados de pesquisas pontuais mostrando a segurança de água fluoretada e dentifrício fluoretado (BEZERRA DE MENEZES et al., 2002; MOYSÉS et al., 2002; PERES et al., 2003; PERES et al., 2009; BARROS; TOMITA, 2010) para o balanço do beneficio anticárie e risco de fluorose que tem sido conseguido no Brasil.

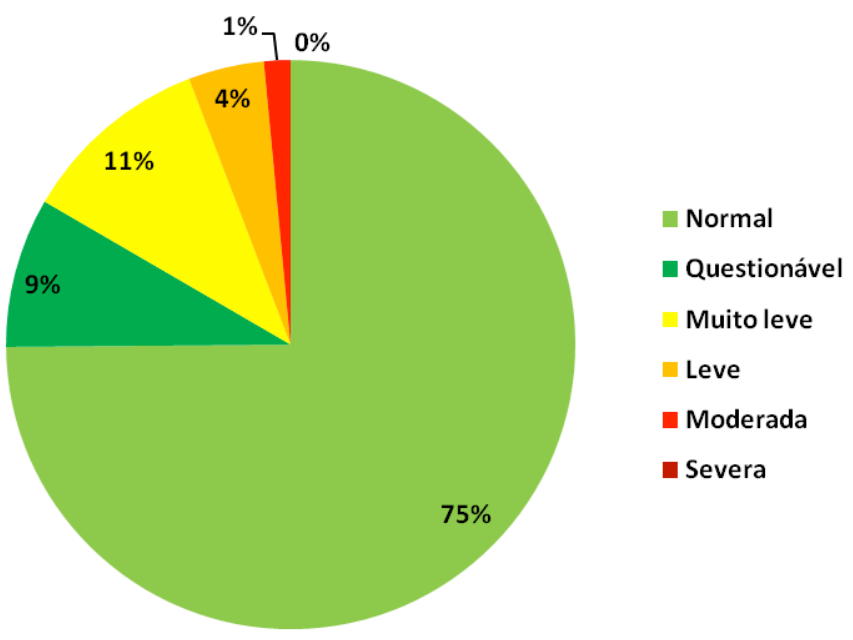

Figura 3: Fluorose dental em adolescentes brasileiros de 25 acordo com o levantamento epidemiológico nacional realizado em 2010 (SB2010).

\section{Considerações Finais}

Quando há mais de 20 anos foi mostrado pela primeira vez que dentifrício fluoretado era fator de risco de fluorose (OSUJI et al., 1988), os autores foram enfáticos ao concluírem que, apesar disso "fluorose dental não era preocupação de saúde pública na cidade onde o trabalho foi realizado". De lá para cá, a avaliação dessa associação não tem sido aprofundada nas dezenas de publicações feitas no mundo inteiro sobre dose de ingestão de fluoreto por crianças e potencial risco de fluorose com base na espúria dose limite de $0,07 \mathrm{mg} \mathrm{F} / \mathrm{dia} / \mathrm{kg}$ de peso, a partir da qual a fluorose seria esteticamente inaceitável.

Nesse artigo, não estamos ignorando o risco de fluorose na $1^{\text {a }}$ infância pelo uso de dentifrício fluoretado, considerando que certa quantidade de fluoreto será ingerida durante as escovações, até as crianças não adquirirem o reflexo de cuspir. Considerando importância do dentifrício fluoretado para o controle de cárie, como discutido nessa série de artigos, o que nos preocupa é a recomendação da postergação até os 3 anos do uso de dentifrício fluoretado, o que não está baseado na melhor evidência disponível (WONG et al., 2010) ou a recomendação de um de baixa concentração para 'evitar' fluorose.

Por outro lado, consideramos que orientar uma criança a usar uma pequena quantidade de creme dental fluoretado é um processo educativo como qualquer outro objetivando criar filhos que possam cuidar de si próprios no futuro, e até que as crianças não possam se cuidar essa tarefa é de responsabilidade dos pais ou cuidadores. Isso sendo feito, estaremos garantindo o balanço entre o beneficio anticárie do fluoreto sem preocupação com fluorose, sendo esse um compromisso de todos aqueles preocupados com saúde publica.

\section{Conclusões}

1. Dentifrício fluoretado é seguro quanto à toxicidade aguda do fluoreto;

2. Dentifrício fluoretado é fator de risco de fluorose dental, mas a dose à qual crianças são submetidas tem sido superestimada;

3. Em região de água fluoretada, fluorose dental não pode ser prevenida pelo uso de dentifrício sem flúor ou de baixa concentração;

4. Em populações expostas a dentifrício fluoretado, a fluorose decorrente não afeta a qualidade de vida dos acometidos, mesmo naquelas já em risco devido à ingestão de flúor pela água.

\section{Referências}

BARROS, B.S.; TOMITA, N.E. Epidemiologic aspects of dental fluorosis in Brazil: research in the period 1993-2006. Cien Saude Colet., v. 15, n. 1, p. 289-300, Jan. 2010.

BEZERRA DE MENEZES, L. M.; SOUSA, M. L. R.; RODRIGUES, L.K.A.; CURY, J. A. Autopercepção da fluorose pela exposição a flúor pela água e dentifrício. Rev. Saúde Pública, São Paulo, v. 36, n.6, p. 752-754, 2002.

BRASIL. Projeto SB Brasil 2010: Resultados principais. Ministério da Saúde, Brasília, DF, 2011.

BRATTHALL, D.; HÄNSEL-PETERSSON, G.; SUNDBERG, $H$. Reasons for the caries decline: what do the experts believe? Eur. J. Oral Sci., v. 104, n. 4 (Pt2); p. 416-422, Aug. 1996. 
BURT, B.A. The changing patterns of systemic fluoride intake. J. Dent. Res., v. 71, Spec. Issue, p. 1228-1237, 1992.

CHANKANKA, O.; LEVY, S.M.; WARREN, J.J.; CHALMERS, J.M. A literature review of aesthetic perceptions of dental fluorosis and relationships with psychosocial aspects/oral health-related quality of life. Community Dent. Oral. Epidemiol., v. 38, n. 2, p. 97-109, Apr. 2010.

COCHRAN, J.A.; KETLEY, C.E.; ARNADÓTTIR, I.B.; FERNANDES, B.; KOLETSI-KOUNARI, H.; OILA, A.M.; VAN LOVEREN, C.; WHELTON, H.P.; O'MULLANE, D.M. A comparison of the prevalence of fluorosis in 8-year-old children from seven European study sites using a standardized methodology. Community Dent. Oral Epidemiol., v. 32, Suppl 1, p. 28-33, Apr. 2004

CONDE, N.C.O.; RABELO, M.A.B.; CURY, J.A. Evaluation of the fluoride stability of dentifrices sold in Manaus, AM, Brazil. Pesqui. Odontol Bras., v. 17, n. 3, p. 247-253, 2003.

CURY, J.A.; TENUTA, L.M.A. Intoxicação aguda por ingestão de flúor. In: ANDRADE, E.A.; RANALI, J. Emergência médicas em Odontologia. 3.ed. São Paulo, Artes Médicas, 2011. Cap. 14, p.145152.

CURY, J.A.; DEL FIOL, F.S.; TENUTA, L.M.A.; ROSALEN, P.L. Lowfluoride dentifrice and gastrointestinal fluoride absorption after meals. J. Dent. Res., v. 84, n.12, p. 1133-1137, 2005.

CURY, J.A.; TENUTA, L.M.; RIBEIRO, C.C.; PAES LEME, A.F. The importance of fluoride dentifrices to the current dental caries prevalence in Brazil. Braz. Dent. J., Ribeirão Preto, v. 15, n. 3, p. 167$174,2004$.

DE ALMEIDA, B.S.; DA SILVA CARDOSO, V.E.; BUZALAF, M.A. Fluoride ingestion from toothpaste and diet in 1- to 3-year-old Brazilian children. Community Dent. Oral Epidemiol., v. 35, n. 1, p. 53-63, Feb. 2007.

DO, L.G.; SPENCER, A.J. Risk-benefit balance in the use of fluoride among young children. J Dent Res., v. 86, n. 8, p. 723-728, Aug. 2007.

DOS SANTOS, A. P.; NADANOVSKY, P.; DE OLIVEIRA, B. H. Inconsistencies in recommendations on oral hygiene practices for children by professional dental and paediatricorganisations in ten countries. Int J Paediatr Dent., v. 21, n. 3, p. 223-251, May 2011.

DOS SANTOS, A.P.; NADANOVSKY, P.; DE OLIVEIRA, B. H. Pesquisa com Secretarias de Saúde no Brasil revela recomendações divergentes relacionadas a práticas de higiene bucal em crianças. Cad. Saúde Pública, Rio de Janeiro?, v. 26, n. 7, p. 14571463, 2010.

DRUMMOND, B.K.; CURZON, M.E.J.; STRONG, M. Estimation of fluoride absorption from swallowed fluoride toothpaste. Caries Res., Basiléia, v. 24, p. 211-215, 1990.

EKSTRAND, J.; SPAK, C.J.; VOGEL, G. Pharmacokinetics of fluoride in man and its clinical relevance. J. Dent. Res., v. 69, p. 550-555, 1990.
EUROPEAN ACADEMY OF PAEDIATRIC DENTISTRY. Guidelines on the use of fluoride in children: an EAPD policy document. Eur. Arch. Paed. Dent., v. 10, n. 3, p. 129-135, 2009.

FALCÃO, A.; TENUTA, L.M.A.; CURY, J.A. Fluoride gastrointestinal absorption from $\mathrm{Na}_{2} \mathrm{FPO}_{3} / \mathrm{CaCO}_{3}$ and $\mathrm{NaF} / \mathrm{SiO}_{2}$-based toothpastes. Caries Res., Basiléia, 2012, aceito.

FEJERSKOV, O.; MANJI, F.; BAELUM, V. The nature and mechanisms of dental fluorosis in man. J. Dent. Res., v. 69, Spec. Issue, p. 692-700, Feb. 1990.

FRAZÃO, P.; PERES, M.A.; CURY, J.A. Drinking water quality and fluoride concentration. Rev. Saude Publica, São Paulo, v. 45, n. 5, p. 964-973, Oct. 2011

MARTINS, C.C.; OLIVEIRA, M.J.; PORDEUS, I.A.; CURY, J.A.; PAIVA, S.M. Association between socioeconomic factors and the choice of dentifrice and fluoride intake by children. Int. J. Environ. Res. Public Health., v. 8, n. 11, p. 4284-4299, Nov. 2011.

MARTINS, C.C.; OLIVEIRA, M.J.; PORDEUS, I.A.; PAIVA, S.M. Comparison between observed children's tooth brushing habits and those reported by mothers. BMC Oral Health. 2011 Sep 3;11:22.

MARTINS, C.C.; PAIVA, S.M.; LIMA-ARSATI, Y.B.; RAMOS-JORGE, M.L.; CURY, J.A. Prospective study of the association between fluoride intake and dental fluorosis in permanent teeth. Caries Res., Basiléia, v. 42, n. 2, p. 125-133, 2008.

MASCARENHAS, A.K. Risk factors for dental fluorosis: a review of the recent literature. Pediatr Dent., v. 22, n. 4, p. 269-277, Jul./Aug. 2000.

MASCARENHAS, A.K.; BURT, B.A. Fluorosis risk from early exposure to fluoride toothpaste. Community Dent. Oral Epidemiol., v. 26, n. 4, p. 241-248, Aug. 1998.

MOYSÉS, S.J.; MOYSÉS, S.T.; ALLEGRETTI, A.C.; ARGENTA, M.; WERNECK, R. Dental fluorosis: epidemiological fiction? Rev. Panam. Salud Publica., v. 12, n. 5, p. 339-346, Nov. 2002.

OSUJI, O.O.; LEAKE, J.L.; CHIPMAN, M.L.; NIKIFORUK, G.; LOCKER, D.; LEVINE, N. Risk factors for dental fluorosis in a fluoridated community. J Dent Res., v. 67, n. 12, p. 1488-1492, Dec. 1988.

PAIVA, S.M.; LIMA, Y.B.O.; CURY, J.A. Fluoride intake by brazilian children from two communities with fluoridated water. Community Dent. Oral. Epidemiol., v. 31, p. 184-191, 2003.

PERES, K.G.; LATORRE, M. DO R.; PERES, M.A.; TRAEBERT, J.; PANIZZI, M. Impact of dental caries and dental fluorosis on 12-yearold schoolchildren's self-perception of appearance and chewing. Cad. Saude Publica, Rio de Janeiro, v. 19, n. 1, p. 323-330, Jan./Feb. 2003.

PERES, K.G.; PERES, M.A.; ARAUJO, C.L.; MENEZES, A.M.; HALLAL, P.C. Social and dental status along the life course and oral health impacts in adolescents: a population-based birth cohort. Health Qual. Life Outcomes, v. 7, p. 95, Nov. 2009. 
RICHARDS, A.; BANTING, D.W. Fluoride toothpastes. In: FEJERSKOV, O.; EKSTRAND, J.; BURT, B.A. Fluoride in Dentistry. 2.ed. Copenhagen: Munksgaard; 1996. p. 328-43.

RICOMINI FILHO, A.P.; TENUTA, L.M.; FERNANDES, F.S.; CALVO, A.F.; KUSANO, S.C.; CURY, J.A. Fluoride concentration in the topselling Brazilian toothpastes purchased at different regions. Braz.

Dent. J., Ribeirão Preto, v. 23, n.1, p. 45-48, 2012.

ROLDI, C.R.; CURY, J.A. Metabolismo do flúor após a ingestão de dentifrício. Rev. Gaúcha Odontol., Porto Alegre, v. 34, p. 425-427, 1986.

TENUTA, L.M.A.; CURY, J.A. Evidências para o uso de fluoretos em Odontologia - Parte III: Limitações do uso de fluoreto em Odontologia - Toxicidade aguda/ Toxicidade crônica (fluorose dental). Jornal da ABO, Ano XXVI, No. 117, Janeiro/Fevereiro 2009, pag. 10-11. http://www.abo.org.br/jornal/117/artigo1.php

WALSH, T.; WORTHINGTON, H. V.; GLENNY, A.- M.; APPELBE, P.; MARINHO, V. C.; SHI, X. Fluoride toothpastes of different concentrations for preventing dental caries in children and adolescents. Cochrane Database Syst Rev 1:CD007868, 2010.

WARREN, J.J.; LEVY, S.M.; BROFFITT, B.; CAVANAUGH, J.E.; KANELLIS, M.J.; WEBER-GASPARONI, K. Considerations on optimal fluoride intake using dental fluorosis and dental caries outcomes - a longitudinal study. J. Public Health Dent., v. 69, p. 1111115, 2009.

WHITFORD, G.M. The metabolism and toxicity of fluoride. Monogr. Oral Sci., v. 16, n. 1-153, 1996.

WHITFORD, G.M.; PASHLEY, D.H. Fluoride absorption: the influence of gastric acidity. Calcif. Tissue Int., v. 36, p. 302-307, 1984.

WHITFORD, G.M. Acute and chronic fluoride toxicity. J. Dent. Res., v. 71, n. 5, p. 1249-1254, May 1992.

WONG, M.C.; GLENNY, A.M.; TSANG, B.W.; LO, E.C.; WORTHINGTON, H.V.; MARINHO, V.C. Topical fluoride as a cause of dental fluorosis in children. Cochrane Database Syst Rev. 1:CD007693, 2010.

Rev. Fac. Odontol. Porto Alegre, v. 53, n. 3, p. 21-27, set./dez., 2012. 